\title{
Improvement of pulmonary dysfunction in patients with severe adult spinal deformity after corrective spinal fusion surgery
}

\author{
Yuki Mihara*1, Tomohiko Hasegawa ${ }^{1}$, Yu Yamato ${ }^{1}$, Go Yoshida ${ }^{1}$, Sho Kobayashi ${ }^{2}$, Tatsuya Yasuda ${ }^{1}$, Tomohiro Banno ${ }^{1}$, \\ Hideyuki Arima ${ }^{1}$, Shin Oe ${ }^{1}$, Hiroki Ushirozako ${ }^{1}$, Tomohiro Yamada ${ }^{1}$, Daisuke Togawa ${ }^{1}$, Yukihiro Matsuyama ${ }^{1}$ \\ ${ }^{1}$ Department of Orthopedic Surgery, Hamamatsu University School of Medicine, Hamamatsu, Japan \\ ${ }^{2}$ Department of Orthopedic Surgery, Hamamatsu Medical Center, Hamamatsu, Japan
}

Received: June 12, 2018

DOI: $10.5430 /$ css.v4n2p15
Accepted: July 26, 2018

Online Published: August 14, 2018

\begin{abstract}
In some cases of adolescent idiopathic scoliosis, corrective surgery can improve pulmonary function. However, the effectiveness of corrective surgery in improving pulmonary function in adult spinal deformity (ASD) has not been reported. Therefore, the purpose of our study was to investigate the recovery of pulmonary function after corrective fusion surgery in 4 patients with severe ASD and associated pulmonary dysfunction. The first patient was a 42-year-old woman with spondylo-epiphyseal dysplasia, whose main presenting complaint was dyspnea. As a result of her respiratory dysfunction, associated with her severe spinal deformity, she required Home Oxygen Therapy (HOT). Prior to surgery, her \%vital capacity (VC) was $25 \%$, with a kyphosis angle of $170^{\circ}$. The second patient was a 55-year-old woman with a history of acromegaly, who presented with low back pain. Prior to surgery, she had a $\% \mathrm{VC}$ of $48 \%$ and a Cobb angle of $85^{\circ}$. The third patient was a 59-year-old woman with adolescent idiopathic scoliosis, which had been previously treated, and who was now experiencing increasing low back pain. Prior to surgery, she had a $\% \mathrm{VC}$ of $58 \%$ and a Cobb angle of $87^{\circ}$. The fourth patient was a 60 -year-old man, with a history of tuberculous spine, who presented with low back pain. Prior to surgery, his $\% \mathrm{VC}$ of $75 \%$ and Cobb angle of $100^{\circ}$. Pulmonary function improved after corrective fusion surgery in all cases. Halo traction with respiratory rehabilitation should be recommended before corrective spinal fusion surgery for patients with severe ASD and pulmonary dysfunction.
\end{abstract}

Key Words: Adult spinal deformity, Pulmonary function, Corrective fusion surgery

\section{INTRODUCTION}

Corrective spinal surgery has been shown to improve pulmonary function in patients with adolescent idiopathic scoliosis, ${ }^{[1-4]}$ with improvements in pulmonary function persisting over a long term. ${ }^{[5,6]}$ However, the effects of corrective spinal surgery on pulmonary function in patients with adult spinal deformity (ASD), as well as in those with pulmonary dysfunction before surgery, have not been evaluated.

Although Bumpass et al. reported pulmonary functions recovered after posterior column resection, the study group included both adult and pediatric patients. ${ }^{[7]}$ Lehman et al. reported a decline in pulmonary function after corrective surgery for ASD. ${ }^{[8]}$ However, patients with pulmonary impairment prior to surgery did experience significant imJapan.

Published by Sciedu Press 
provement in pulmonary function after corrective surgery. Therefore, the purpose of this case series report was to investigate the effect of corrective spinal surgery on the pulmonary function in 4 patients with severe ASD and associated respiratory dysfunction, wherein pulmonary dysfunction was defined as a percent vital capacity (\%VC) of $<80 \%$.

Pulmonary function tests, thoracic computed tomography (CT) and whole spine standing radiography were performed at the first visit (baseline before surgery), at the postoperative follow-up, and at the final follow-up. VC and the percent forced expiratory volume in one second (FEV1.0\%) were used to evaluate pulmonary function. Thoracic capacity was calculated from thoracic CT data using the Synapse Vincent software, as per previously described methods. ${ }^{[9]}$ The Cobb angle and the angle of spinal kyphosis were measured from the standing radiographs. ${ }^{[10]}$

\section{Case presentations}

\subsection{Case 1}

The patient was a 42-year-old woman with spondyloepiphyseal dysplasia, whose main complaint on presentation was dyspnea. She had undergone corrective surgery when she was 8 years old at another hospital, with revision surgery performed due to rod fracture when she was 9 years old. She began experiencing dyspnea, due to respiratory dysfunction associated with her severe spinal deformity, when she was 39 years old. After a year of progressive dyspnea, Home Oxygen Therapy (HOT) was initiated. Moreover, a year prior to her first consultation with us, she suffered from heart failure associated with her spinal deformity. The neurological examination was unremarkable. On standing radiographs, a kyphoscoliosis deformity was clearly observable, with a kyphotic angle of $170^{\circ}$; the deformity was too severe to measure the Cobb angle in the coronal plane (see Figure 1A and 1B).

Considering the known natural history of this type of spinal deformity, corrective fusion surgery was indicated. The $\% \mathrm{VC}, \mathrm{VC}, \mathrm{FEV} 1.0 \%$, and thoracic volume, measured at the first baseline visit, were $25 \%, 370 \mathrm{ml}, 87 \%$, and $729 \mathrm{ml}$, respectively (see Table 1 ). We deemed the severity of her respiratory dysfunction was prohibitive of surgical intervention. Therefore, we proceeded with application of halo traction and respiratory rehabilitation. Over the 12 weeks of hospitalization, her VC gradually improved from $34 \%$ to $41 \%$. After this intensive period, the patient continued respiratory rehabilitation for another 2 years, on an out-patient status. Over this time, her \%VC, VC, FEV1.0\%, and thoracic volume improved to $44 \%, 640 \mathrm{ml}, 91 \%$, and $942 \mathrm{ml}$, respectively (see Table 1), and corrective spinal fusion surgery was planned.

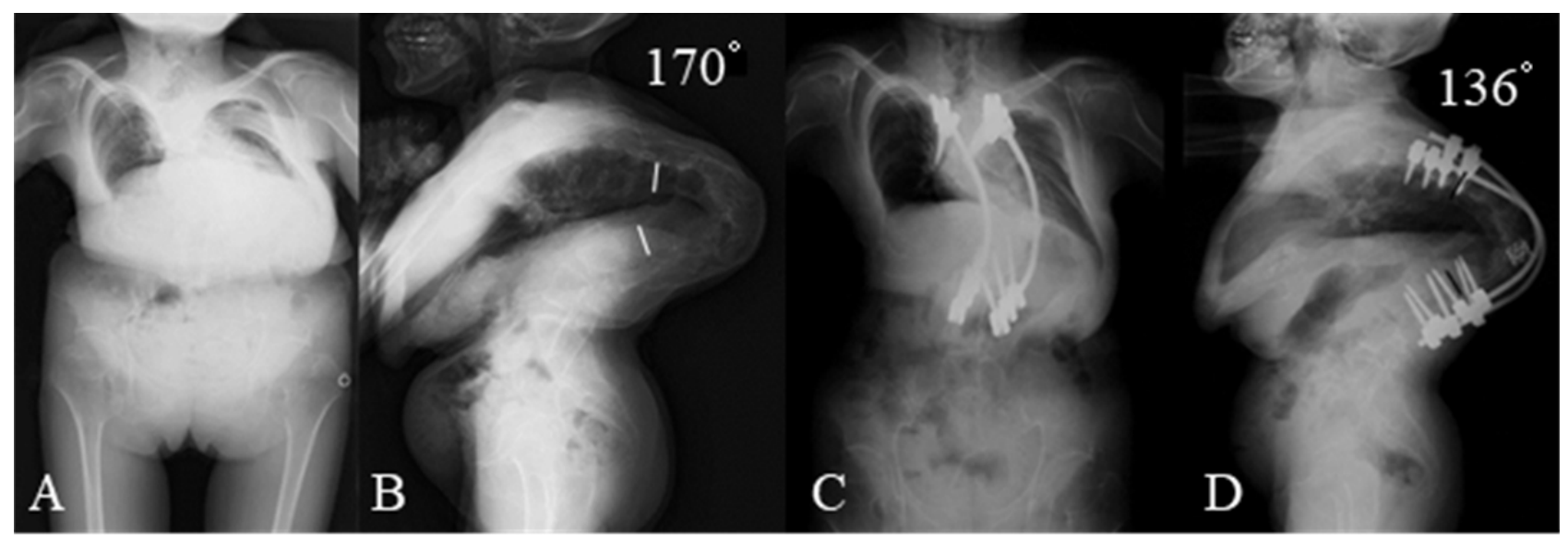

Figure 1. Pre-operative antero-posterior (A) and lateral (B) whole spine standing radiograph, with the $170^{\circ}$ angle of kyphosis identified. Postoperative antero-posterior (C) and lateral (D) whole spine standing radiograph, with the $136^{\circ}$ angle of kyphosis identified. The correction rate was $20 \%$.

Table 1. Time course of each parameters in case 1

\begin{tabular}{llll}
\hline & First visit & Pre-Op & Post-Op 2Y \\
\hline$\%$ VC $(\%)$ & 25 & 44 & 41 \\
VC (ml) & 370 & 640 & 690 \\
FEV1.0\% (\%) & 87 & 91 & 82 \\
Thoracic volume (ml) & 729 & 942 & 1,305 \\
\hline
\end{tabular}

The surgery was performed in 2 steps. During the first surgery, we explored the deformity of the spine and placed the pedicle screws under navigation, from T3 to L4, without osteotomy. During the second surgery, performed 8 months later, we proceeded with posterior vertebral column resection (PVCR), from T10-T12. The kyphosis angle decreased from $170^{\circ}$ to $136^{\circ}$ postoperatively (see Figure 1C and 1D). 
The correction rate was $20 \%$. Two years after surgery, her VC and the thoracic volume improved further to $690 \mathrm{ml}$ and 1,305 $\mathrm{ml}$, respectively (see Table 1 ).

\subsection{Case 2}

The patient was a 55-year-old woman with a history of acromegaly, who presented with low back pain. She underwent tumorectomy of a pituitary tumor at the age of 33 years. She experienced increased low back pain at the age of 47 years, for which she underwent a 4-year course of conservative treatment. However, due to progression of her spinal deformity and dyspnea, corrective surgery was planned at the previous hospital when she was 51 years old. However, this surgery was canceled when she developed $\mathrm{CO}_{2}$ narcosis. After another delay of 4 years, she was referred to our department for corrective surgery. Her neurological examination was unremarkable. The Cobb angle, measured on standing radiographs, was $85^{\circ}$, with a kyphosis angle of $89^{\circ}$ (see Figure $2 \mathrm{~A}$ and $2 \mathrm{~B})$. Her \%VC, VC, FEV1.0\%, and thoracic volume at baseline were $48 \%, 1,050 \mathrm{ml}, 89 \%$, and $1,637 \mathrm{ml}$, respectively. Corrective fusion surgery was planned, extending from the upper thoracic spine to the pelvis. Because of the extent the patient's respiratory dysfunction, a MIS approach was recommended by the anesthesiologist, pre-operatively.

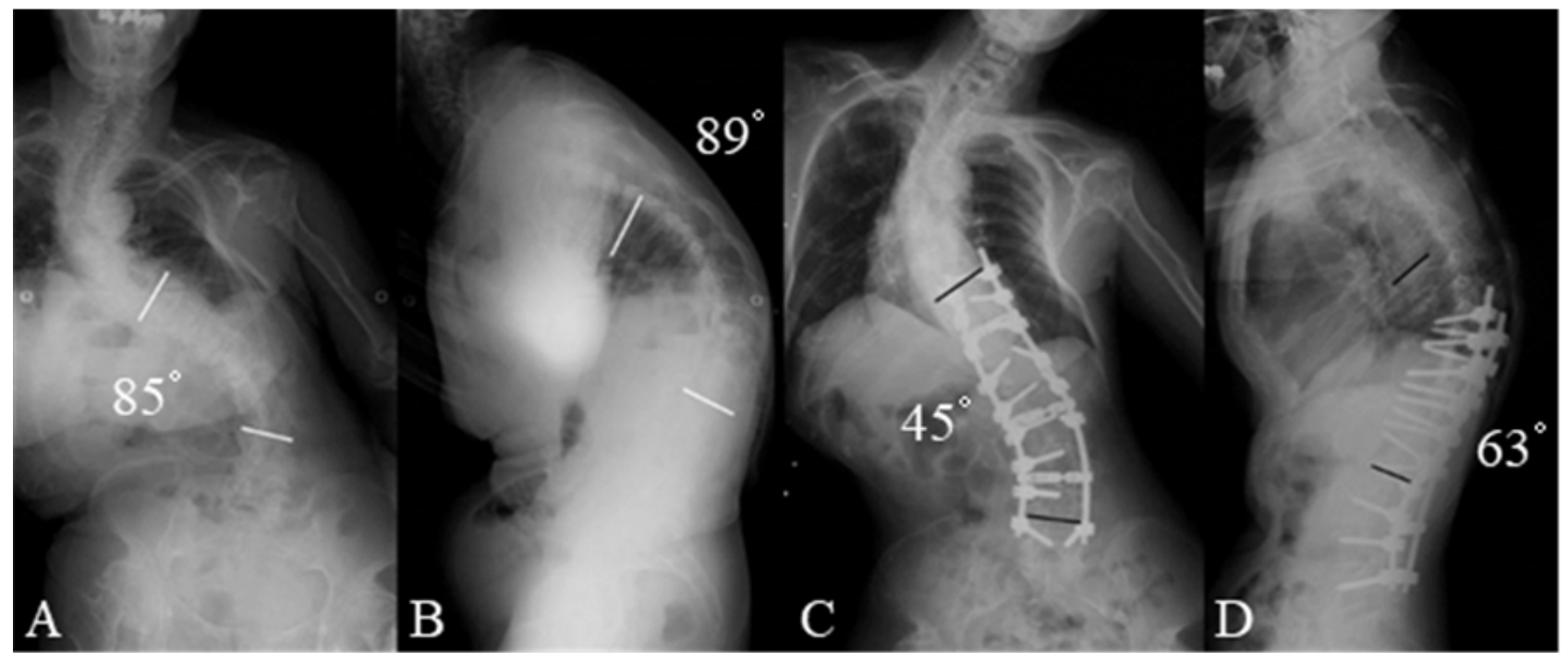

Figure 2. Pre-operative antero-posterior (A) and lateral (B) whole spine standing radiograph, with the $85^{\circ} \mathrm{Cobb}$ angle and $89^{\circ}$ angle of kyphosis identified. Postoperative antero-posterior (C) and lateral (D) whole spine standing radiograph, with the $45^{\circ} \mathrm{Cobb}$ angle and $63^{\circ}$ angle of kyphosis identified. The correction rates were $41 \%$ and $26 \%$, respectively.

We proceeded with a posterior fusion surgery, from T9 to L5. Postoperatively, her Cobb angle decreased from $85^{\circ}$ to $50^{\circ}$, with a decrease in her kyphosis angle from $89^{\circ}$ to $63^{\circ}$ (see Figure 2C and 2D). The correction rates were $41 \%$ and $26 \%$, respectively. Her \% VC and VC increased to $54 \%$ and $1,140 \mathrm{ml}$, respectively, 6 months after surgery. However, the patient developed repeated pneumonia after surgery, and her $\% \mathrm{VC}$ and VC subsequently decreased to $39 \%$ and $900 \mathrm{ml}$, respectively, at 4 years after surgery, but with no effect on the thoracic volume, which was $2,044 \mathrm{ml}$ at 4 years after surgery (see Table 2).

Table 2. Time course of each parameters in case 2

\begin{tabular}{llll}
\hline & Pre-Op & Post-Op 6M & Post-Op 4Y \\
\hline \%VC (\%) & 48 & 54 & 39 \\
VC (ml) & 1,050 & 1,140 & 900 \\
FEV1.0\% (\%) & 89 & 86 & 98 \\
Thoracic volume (ml) & 1,637 & - & 2,044 \\
\hline
\end{tabular}

Published by Sciedu Press

\subsection{Case 3}

A 59-year-old woman with adolescent idiopathic scoliosis, which had been treated during her high-school years, presented with a 3-year history of low back pain. Her neurological examination was unremarkable. The Cobb angle was $87^{\circ}$, with a kyphosis angle of $86^{\circ}$ (see Figure $3 \mathrm{~A}$ and 3B). Her \% VC, VC, FEV1.0\%, and thoracic volume at baseline were $58 \%, 1,600 \mathrm{ml}, 99 \%$, and 3,122 $\mathrm{ml}$, respectively. Posterior fusion surgery was performed from T5 to the iliac crests. Postoperatively, her Cobb angle decreased to $53^{\circ}$ and the angle of kyphosis, to $62^{\circ}$ (see Figure $3 \mathrm{C}$ and $3 \mathrm{D}$ ). The correction rates were $39 \%$ and $28 \%$, respectively. Her $\% \mathrm{VC}, \mathrm{VC}, \mathrm{FEV} 1.0 \%$, and thoracic volume were maintained at $58 \%, 1,640 \mathrm{ml}, 96 \%$, and $3,113 \mathrm{ml}$, respectively, at 1 year after surgery. These pulmonary function values subsequently improved to $64 \%, 1,790 \mathrm{ml}, 98 \%$, and 3,315 ml, respectively, at 2 years after surgery (see Table 3 ). 


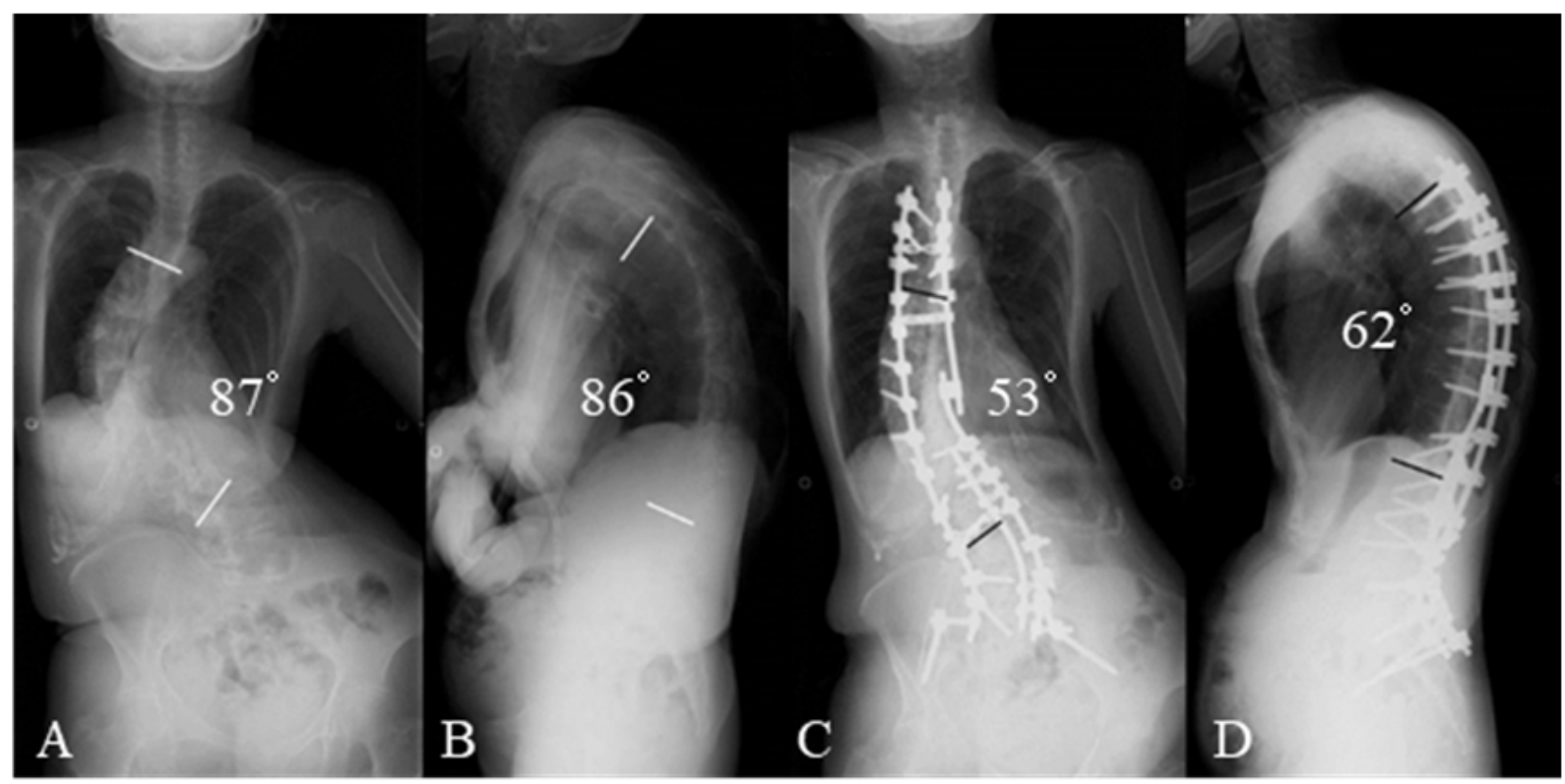

Figure 3. Pre-operative antero-posterior (A) and lateral (B) whole spine standing radiograph, with the Cobb angle of $87^{\circ}$ and angle of kyphosis of $86^{\circ}$ identified. Postoperative antero-posterior (C) and lateral (D) whole spine standing radiograph, with the Cobb angle of $53^{\circ}$ of Cobb and angle of kyphosis of $62^{\circ}$ identified. The correction rates were $39 \%$ and $28 \%$, respectively.

Table 3. Time course of each parameters in case 3

\begin{tabular}{llll}
\hline & Pre-Op & Post-Op 1Y & Post-Op 2Y \\
\hline$\%$ VC (\%) & 58 & 58 & 64 \\
VC (ml) & 1,600 & 1,640 & 1,790 \\
FEV1.0\% (\%) & 99 & 96 & 98 \\
Thoracic volume (ml) & 3,122 & 3,113 & 3,315 \\
\hline
\end{tabular}

Table 4. Time course of each parameters in case 4

\begin{tabular}{lll}
\hline & Pre-Op & Post-Op 6Y \\
\hline$\% \mathrm{VC}(\%)$ & 75 & 80 \\
$\mathrm{VC}(\mathrm{ml})$ & 2,400 & 2,630 \\
FEV1.0\% (\%) & 75 & 79 \\
Thoracic volume (ml) & 2,647 & 3,443 \\
\hline
\end{tabular}

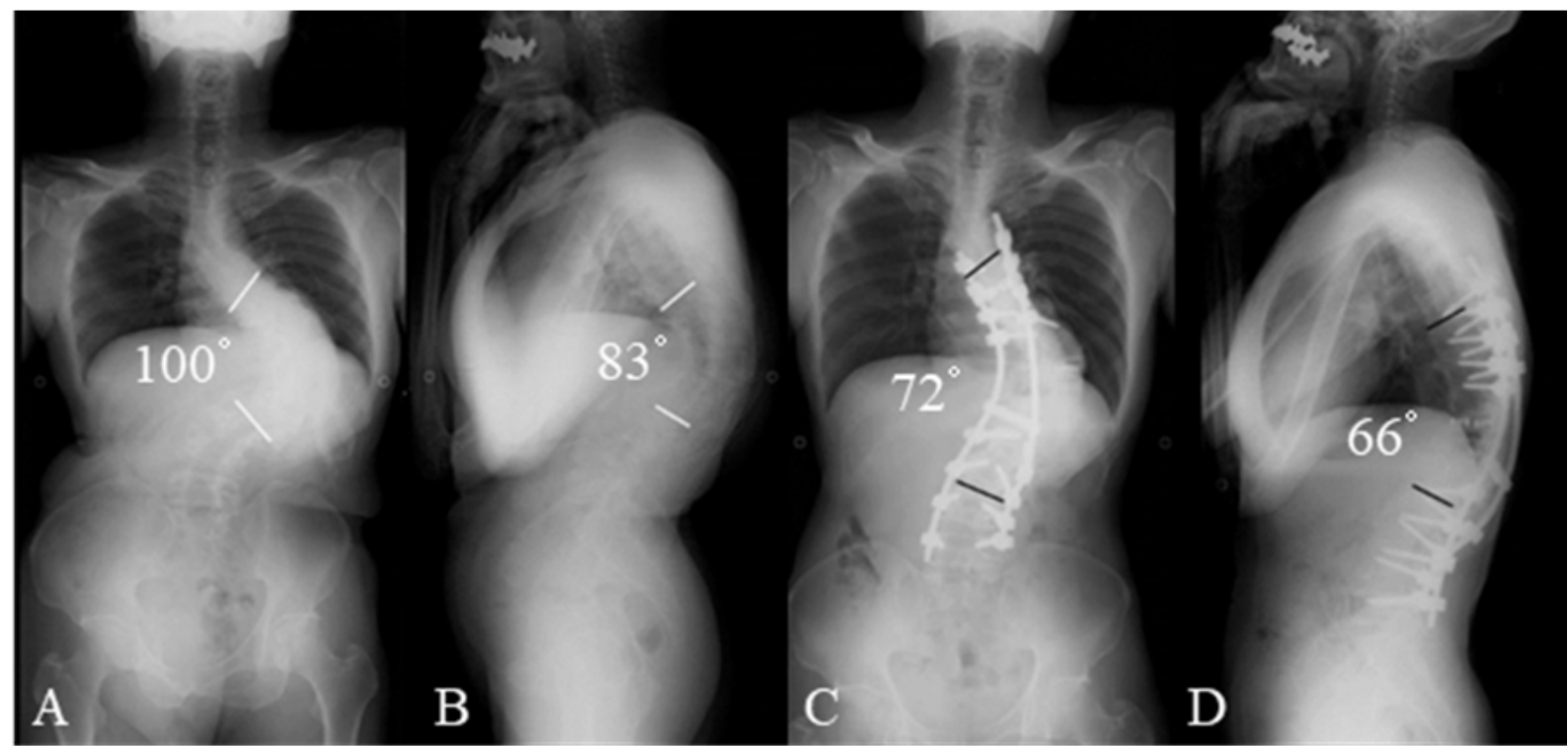

Figure 4. Pre-operative antero-posterior (A) and lateral (B) whole spine standing radiograph, with the Cobb angle of $100^{\circ}$ and angle of kyphosis of $83^{\circ}$ indicated. Postoperative antero-posterior (C) and lateral (D) whole spine standing radiograph, with the Cobb angle of $72^{\circ}$ and angle of kyphosis of $66^{\circ}$ indicated. The correction rates were $28 \%$ and $20 \%$, respectively. 


\subsection{Case 4}

The patient was a 60-year-old man, with a history of tuberculous spine from his elementary school years, who presented with a 5-year history of low back pain. The neurological examination was unremarkable. The Cobb angle was $100^{\circ}$, with an angle of kyphosis of $83^{\circ}$ (see Figure 4A and 4B). His $\% \mathrm{VC}, \mathrm{VC}, \mathrm{FEV} 1.0 \%$, and thoracic volume at baseline were $75 \%, 2,400 \mathrm{ml}, 75 \%$, and $2,647 \mathrm{ml}$, respectively. A PVCR of T11 was performed to correct the spinal deformity, with spinal fusion from T6 to L5. Postoperatively, the Cobb angle decreased to $72^{\circ}$ and the angle of kyphosis to $66^{\circ}$ (see Figure $4 \mathrm{C}$ and $4 \mathrm{D}$ ). The correction rates were $28 \%$ and $20 \%$, respectively. His \% VC, VC, FEV1.0\%, and thoracic volume improved to $80 \%, 2,630 \mathrm{ml}, 99 \%$, and 3,443 $\mathrm{ml}$, respectively, at 6 year after surgery (see Table 4 ).

\section{Discussion}

The natural course of severe adult ASD includes respiratory dysfunction, which inevitably results, over time, in pulmonary heart disease and pneumonia. ${ }^{[1,12]}$ Whether corrective surgery can improve pulmonary function in these patients is an issue of controversy, with some studies reporting little improvement; ${ }^{[7,8,10]}$ however, Lehman et al. reported improvement in patients with ASD who had respiratory dysfunction before surgery. ${ }^{[8]}$ Our case series study supports the finding of Lehman et al., with findings of improved pulmonary function in all 4 patients with severe ASD, all of whom had pulmonary dysfunction before surgery. We also demonstrate that respiratory rehabilitation was effective in the case of a 42-year-old woman who was experiencing dyspnea because of severe spinal deformity due to spondyloepiphyseal dysplasia.

Fuschillo et al. also reported that respiratory rehabilitation was effective in patients with severe respiratory impairment associated with kyphoscoliosis. ${ }^{[13]}$ In our case, we applied halo traction which was effective in providing us the time needed to pursue the respiratory rehabilitation and improve the patient's pulmonary function to the level needed to proceed to spinal surgical correction and fusion, as per previously published approaches for correction of severe scoliosis with cor pulmonale and severe ASD with respiratory failure $^{[14,15]}$ Based on previous report, patient with cor pulmonale due to spinal deformity should have a trial of halo traction before operations. If their pulmonary functions improve, then surgical stabilization can be performed. ${ }^{[14]}$ In severe ASD patients with respiratory failure, pre-operative halo traction was also effective to estimate their post-operative prognosis. ${ }^{[15]}$ In our case, the combination of 12-week program of respiratory rehabilitation and halo traction improved the patient's \% VC from $34 \%$ to $41 \%$. This extent of improvement was comparable to the $27.3 \%$ (range, $17 \%-50 \%$ ) improvement in \% VC after an average respiratory rehabilitation of 3.7 week (range, 1-24 weeks) reported by Rizzi et al. ${ }^{[15]}$ From the perspective of anesthesiology, a $\% \mathrm{VC}$ of $<$ $50 \%$ increases the risk of postoperative morbidity and mortality. ${ }^{[16]}$ Therefore, a $\% \mathrm{VC}$ of $<50 \%$ might be one cut off value to for the use of halo traction and respiratory rehabilitation before surgical correction. Considering these results, we propose that hallo traction and pre-operative respiratory rehabilitation could have been beneficial in improvement clinical outcomes in patient 2 , whose postoperative $\% \mathrm{VC}$ initially improved postoperatively but subsequently decreased due to repeated pneumonia.

Pulmonary function improved after corrective fusion surgery in patients with severe ASD associated with pulmonary dysfunction. Halo traction with respiratory rehabilitation should be recommended, before corrective spinal fusion surgery for patients with severe ASD and pulmonary dysfunction.

\section{CONTRIBUTORS}

(I) Conception and design: Y Mihara and Y Matsuyama; (II) Administrative support: None; (III) Provision of study materials or patients: All authors; (IV) Collection and assembly of data: All authors; (V) Data analysis and interpretation: All authors; (VI) Manuscript writing: All authors; (VII) Final approval of manuscript: All authors.

\section{CONFLICTS OF INTEREST Disclosure}

Shin Oe and Daisuke Togawa belong to a donated fund laboratory known as the Division of Geriatric Musculoskeletal Health.

\section{REFERENCES}

[1] Kim YJ, Lenke LG, Bridwell KH, et al. Prospective pulmonary function comparison following posterior segmental spinal instrumentation and fusion of adolescent idiopathic scoliosis: is there a relationship between major thoracic curve correction and pulmonary function test improvement? Spine. 2007; 32(24): 2685-93.
[2] Kovac V, Puljiz A, Smerdelj M, et al. Scoliosis curve correction, thoracic volume changes, and thoracic diameters in scoliotic patients after anterior and after posterior instrumentation. International Orthopaedics. 2001; 25(2): 66-9. PMid: 11409453. https: //doi.org/10.1007/s002640100233

[3] Vedantam R, Lenke LG, Bridwell KH, et al. A prospective evaluation 
of pulmonary function in patients with adolescent idiopathic scoliosis relative to the surgical approach used for spinal arthrodesis. Spine. 2000; 25(1): 82-90. PMid: 10647165. https ://doi.org/10.109 7/00007632-200001010-00015

[4] Wood KB, Schendel MJ, Dekutoski MB, et al. Thoracic volume changes in scoliosis surgery. Spine. 1996; 21(6): 718-23. PMid: 8882694. https ://doi.org/10.1097/00007632-19960 3150-00012

[5] Gitelman Y, Lenke LG, Bridwell KH, et al. Pulmonary function in adolescent idiopathic scoliosis relative to the surgical procedure: a 10-year follow-up analysis. Spine. 2011; 36(20): 1665-72.

[6] Pehrsson K, Danielsson A, Nachemson A. Pulmonary function in adolescent idiopathic scoliosis: a 25 year follow up after surgery or start of brace treatment. Thorax. 2001; 56(5): 388-93. PMid: 11312408 https://doi.org/10.1136/thorax.56.5.388

[7] Bumpass DB, Lenke LG, Bridwell KH, et al. Pulmonary function improvement after vertebral column resection for severe spinal deformity. Spine. 2014; 39(7): 587-95.

[8] Lehman RA, Jr., Kang DG, Lenke LG, et al. Pulmonary function following adult spinal deformity surgery: minimum two-year followup. The Journal of Bone and Joint Surgery American Volume. 2015; 97(1): 32-9.

[9] Kitano M, Iwano S, Hashimoto N, et al. Lobar analysis of collapsibility indices to assess functional lung volumes in COPD patients.
International Journal of Chronic Obstructive Pulmonary Disease. 2014; 9: 1347-56.

[10] Zeng Y, Chen Z, Ma D, et al. The influence of kyphosis correction surgery on pulmonary function and thoracic volume. Spine. 2014; 39(21): 1777-84.

[11] McMaster MJ, Glasby MA, Singh H, et al. Lung function in congenital kyphosis and kyphoscoliosis. Journal of Spinal Disorders \& Techniques. 2007; 20(3): 203-8.

[12] Satoh F, Fujita MQ, Seto Y, et al. Sudden death in a patient with idiopathic scoliosis. Journal of Clinical Forensic Medicine. 2006; 13(6-8): 335-8.

[13] Fuschillo S, De Felice A, Martucci M, et al. Pulmonary rehabilitation improves exercise capacity in subjects with kyphoscoliosis and severe respiratory impairment. Respiratory Care. 2015; 60(1): 96-101.

[14] Swank SM, Winter RB, Moe JH. Scoliosis and cor pulmonale. Spine. 1982; 7(4): 343-54. PMid: 7135067. https ://doi.org/10.109 7/00007632-198207000-00004

[15] Rizzi PE, Winter RB, Lonstein JE, et al. Adult spinal deformity and respiratory failure. Surgical results in 35 patients. Spine. 1997; 22(21): 2517-30; discussion 31. PMid: 9383859. https ://doi.or g/10.1097/00007632-199711010-00011

[16] Gass GD, Olsen GN. Preoperative pulmonary function testing to predict postoperative morbidity and mortality. Chest. 1986; 89(1): 127-35. https ://doi.org/10.1378/chest.89.1.127 